\title{
Difficulty in predicting intra-abdominal adhesion before cesarean section: A case report
}

\author{
Nanao Suzuki ${ }^{1}$, Yu Wakaki ${ }^{1}$, Kaori Watanabe ${ }^{1}$, Yukiko Kumasaka ${ }^{1}$, and Rika Suzuki ${ }^{1}$ \\ ${ }^{1}$ Ohara General Hospital
}

January 3, 2022

\begin{abstract}
Adhesions between the bladder and uterus necessitated an atypical incision in the cesarean section of a woman with endometriosis. This could not be predicted with pre-surgery MRI. .No methods in the literature are able to predict adhesions with true certainty; it is therefore still difficult to diagnose intra-abdominal adhesions

Difficulty in predicting intra-abdominal adhesion before cesarean section: A case report

Nanao Suzuki ${ }^{1}$, Yu Wakaki ${ }^{1}$, Kaori Watanabe ${ }^{1}$, Yukiko Kumasaka ${ }^{2}$, Rika Suzuki ${ }^{1}$,

${ }^{1}$ Department of Obstetrics and Gynecology, ${ }^{2}$ Department of Radiology, Ohara General Hospital, Fukushima, Japan

Corresponding author: Nanao Suzuki

Department of Obstetrics and Gynecology, Ohara General Hospital, 6-1 Uwamachi, Fukushima 960-8611, Japan

Tel.: +81-24-526-0300, Fax: +81-24-526-0342, E-mail: nanao77@fmu.ac.jp

Coauthor: Yu Wakaki

Department of Obstetrics and Gynecology, Ohara General Hospital, 6-1 Uwamachi, Fukushima 960-8611, Japan

Tel.: +81-24-526-0300, Fax: +81-24-526-0342, E-mail: y-wakaki@ohara-hp.or.jp

Coauthor: Kaori Watanabe

Department of Obstetrics and Gynecology, Ohara General Hospital, 6-1 Uwamachi, Fukushima 960-8611, Japan

Tel.: +81-24-526-0300, Fax: +81-24-526-0342, E-mail: kwtnb@ohara-hp.or.jp

Coauthor: Yukiko Kumasaka

Department of Radiology, Ohara General Hospital, 6-1 Uwamachi, Fukushima 960-8611, Japan

Tel.: +81-24-526-0300, Fax: +81-24-526-0342, E-mail: y-wakaki@ohara-hp.or.jp

Coauthor: Rika Suzuki

Department of Obstetrics and Gynecology, Ohara General Hospital, 6-1 Uwamachi, Fukushima 960-8611, Japan
\end{abstract}


Tel.: +81-24-526-0300, Fax: +81-24-526-0342, E-mail: rikamaru@ohara-hp.or.jp

\section{Funding}

This research did not receive any specific grant from funding agencies in the public, commercial, or not-forprofit sectors.

\section{Conflict of Interest}

The authors have no potential conflicts of interest to declare.

\section{Patient Consent}

Written informed consent was obtained from the patient for the publication of this work

\section{Abstract}

Severe adhesions between the bladder and uterus necessitated an atypical incision in the cesarean section of a woman with endometriosis. This could not be predicted with pre-surgery MRI. No methods in the literature are able to predict adhesions with true certainty; it is therefore still difficult to diagnose intra-abdominal adhesions.

\section{Introduction}

The lower transverse incision is a basic surgical technique for cesarean sections, but other uterine incisions may be selected in certain cases, such as placenta previa and accreta. We present a case of fundal uterine incision delivery due to severe adhesions between the bladder and uterus caused by endometriosis. There is no description of such adhesion caused by endometriosis in the recent literature review regarding difficult cesarean sections by Visconti et al. ${ }^{1}$ Moreover, we could not find a report that clarifies the provides solutions to the difficulties of performing cesarean surgery in women with endometriosis; hence, we could not predict abdominal adhesions or uterine displacement by magnetic resonance imaging (MRI) before delivery. As a consequence, we searched for methods predicting intra-abdominal adhesions preoperatively.

Case

A 41-year-old nulliparous woman diagnosed with endometriosis during treatment for infertility was referred to our hospital at 13 weeks of gestation after in vitro fertilization (IVF) and frozen embryo transfer (FET). She was prescribed low-dose estrogen progestin pills before the IVF-FET. We suspected placenta previa based on transvaginal ultrasound findings at 24 weeks of gestation and diagnosed the patient with total placenta previa at 30 weeks of gestation by MRI (Fig. 1a). Massive genital bleeding and uterine contractions appeared at 31 weeks of gestation. The patient was hospitalized and prescribed magnesium sulfate intravenously (1.0 $\mathrm{g}$ per hour) and antenatal corticosteroids (betamethasone $12 \mathrm{mg}$ twice every 24 hours) intramuscularly. The symptoms recurred four days later, and intravenous ritodrine hydrochloride was administered. Since tocolysis was infeasible due to the side effects of the beta-mimetic agent, including parotid gland swelling and elevated serum amylase $(3074 \mathrm{U} / \mathrm{L})$, we performed a cesarean section under spinal anesthesia.

After a lower vertical abdominal incision, the right round ligament and oviduct were located in the center of the surgical field, and there were firm adhesions between the bladder and the anterior uterine wall. We made a midline vertical (classical) incision to avoid bladder injury, and a male infant weighing $1884 \mathrm{~g}$ was delivered in the cephalic position, with Apgar scores of 3 and 8. The infant was admitted to the Neonatal Intensive Care Unit (NICU) due to prematurity and low birth weight. The placenta was placed on the internal ostium of the uterus and was separated smoothly without massive bleeding. After the uterine suture, the incision was found to cross through the uterine fundus toward the posterior wall (Fig. 2). Consequently, the incision was similar to a transverse fundal uterine incision. We also found lesions of endometriosis, and adhesions between the posterior wall of the uterus and the sigmoid colon (Fig. 3). Anti-adhesion agents were placed on the fundal uterine incision, and the abdominal wall was sutured. The estimated blood loss, including amniotic fluid during surgery, was $1605 \mathrm{~mL}$, and the operation time was $56 \mathrm{~min}$. 
One day post-surgery, the patient's hemoglobin level was $9.2 \mathrm{~g} / \mathrm{dL}$; blood transfusion was therefore avoided. The mother was discharged six days after surgery. At 41 days postpartum, we found a hyperechoic lesion, representing the wound, located in the uterine fundus (Fig. 4). The baby was discharged from the NICU 60 days after birth, without any major complications.

\section{Discussion}

Since the placenta covered the internal uterine ostium in the MRI image at 30 weeks gestation, we diagnosed the patient with placenta previa (Fig. 1a). The bladder, anterior, fundus, and posterior uterine walls were located towards the front of the body (Figure 1b). This situation was not predicted upon preoperative MRI. Intraoperative findings revealed that the uterus was displaced, and the bilateral round ligaments and oviducts were located in towards the front (Fig. 2). The adhesions between the bladder and uterus may have resulted from endometriosis. The patient demonstrated foci at the closed Douglas pouch during the operation (Fig. 2 and 3). Endometriosis was observed only by inspection and was not confirmed by pathological examination. However, the diagnosis was assumed to be correct because there was no history of pelvic inflammatory disease or abdominal surgery. The obstetricians and radiologists of the team retrospectively discussed the prenatal MRI during the postpartum period; however, they could not detect the round ligament or oviduct in the image because of the resolving limit. We investigated whether there were other methods available to diagnose unusual uterine positions.

\section{Difficult Cesarean Sections}

Visconti et al. reviewed difficult cesarean sections. ${ }^{1}$ The article discussed "difficult" cesarean sections divided into four categories: difficult access to the lower uterine segment, complicated fetal extraction, laceration or organ damage, and abnormal placentation. The "difficult access" category included leiomyomas, obesity, and previous abdominal surgery, but not endometriosis. The article stated that the degree of adhesions created after abdominal surgery varies widely among individuals, making it impossible to predict. Therefore, if we are unable to distinguish between endometriosis and postoperative adhesions, adhesions caused by endometriosis are also considered difficult to predict. Previous studies have reported several methods that may predict intra-abdominal adhesions.

\section{Abdominal Scar Characteristics}

There are several reports on whether differences in the color and shape of skin markers and striae gravidarum can predict intra-abdominal adhesions in pregnant women who have had at least one prior surgery. Prospective comparative studies have reported that factors related to skin markers, such as scar color and length, are associated with intra-abdominal adhesions. ${ }^{2-4}$ In contrast, Taylan et al. denied the accuracy of predictions using these methods, ${ }^{5}$ and Jaafer et al. demonstrated that these markers are not clinically reliable. $^{6}$

\section{Sliding Sign}

Reid et al. presented the 'sliding sign' technique to predict a closed pouch of Douglas preoperatively. ${ }^{7,8}$ The technique using transvaginal ultrasonography is well known as a non-invasive and effective approach for detecting endometriotic adhesions in the pouch of Douglas and deep infiltrating endometriosis. A negative 'sliding sign' was noted when the anterior rectosigmoid colon or the anterior rectum was fixed to the posterior uterine fundus or retrocervix. Hudelist et al. also concurred with these findings and deemed them useful. ${ }^{9}$ Ichikawa et al. proposed a scoring system that allowed for an accurate prediction of pelvic adhesion status and may potentially be an indicator of postoperative adhesion and infertility. ${ }^{10}$

However, all these examinations were performed in a non-pregnant state; hence, it is doubtful whether they are useful for predicting adhesions of the anterior wall of the uterus in pregnant women, as in this case.

Baron et al. examined the 'sliding sign' of the uterus under the inner part of the fascia of the abdominal muscles during deep breathing in the third trimester. ${ }^{11}$ They reported that it was useful for predicting the 
presence or absence of intra-abdominal adhesions. This method may be useful for this case; however, a report of 112 pregnant women in 2021 revealed low reproducibility of these results. ${ }^{12}$

\section{MRI}

Some articles suggested that MRI cannot be used for definitive diagnosis or endometriosis staging. ${ }^{13,14}$ Therefore, laparoscopy remains the procedure of choice. MRI has a high sensitivity for the diagnosis of ovarian endometriosis, but it has poor results in the detection of other types of endometrioses, including intra-abdominal adhesions. Randall et al. reported that the 'sheargram,' cine-MRI technique depicts the amount of sliding between the abdominal contents and the wall of the abdominal cavity during respiratory cycles ${ }^{15,16}$ but the results have yet to be generalized.

In this case, MRI performed during pregnancy showed a raised bladder, but no intra-abdominal adhesions. Therefore, we concluded that adhesions are difficult to predict using MRI alone. Since our patient also had adhesions in the pouch of Douglas, it may be possible that the 'sliding sign' of the posterior fornix and adhesions between the abdominal wall and uterus could be detected. However, a large clinical study of the 'sliding sign' technique does not exist and therefore should be a topic for future research.

Fundal Uterine Incision

Kotsuji et al. reported a case of transverse fundal uterine incision in $2004,{ }^{17}$ and a case series in $2014,{ }^{18}$ which showed that this procedure has the potential to avoid transection of the placenta, preventing heavy bleeding and catastrophic fetal blood loss. However, such a case is rare, and the actual risk of uterine rupture and placenta accreta in subsequent pregnancies is unknown. In our case, the anterior uterine wall adhered to the vesicouterine pouch. We cut open the uterine corpus to create a classical incision, while avoiding bladder damage; however, a transverse fundal uterine incision was made, which may result in an increased risk of uterine rupture and placenta accreta in subsequent pregnancies.

Endometriosis and Pregnancy Outcome

It has been demonstrated that women with a history of endometriosis have an increased risk of obstetric complications, such as placenta previa, preterm delivery, preterm premature rupture of membranes, and stillbirth and the severity of endometriosis may have an adverse impact on pregnancy outcomes. ${ }^{19-21}$ This case could be evaluated as stage IV in the revised American Society for Reproductive Medicine scoring system; the case has a high-risk for obstetrical complications ${ }^{20}$ that may develop into placenta previa and preterm delivery. Firm adhesion caused an atypical uterine incision. Several methods for predicting intra-abdominal adhesions before surgery have been reported, and it is necessary to use such procedures. However, it is difficult to diagnose all conditions in the abdominal cavity. Physicians should consider the possibility of an unusual uterine incision in women with a history of endometriosis, adenomyosis, or severe dysmenorrhea. It is also necessary to share information with the surgical team in charge and prepare for possible damage to the surrounding organs. Advances in and increased utilization of assisted reproductive technology has resulted in an increased rate of pregnancies with severe endometriosis, which means that unexpected complications can occur during prenatal and delivery periods.

\section{Conclusion}

We encountered a case where the anterior wall of the uterus adhered to the vesicouterine pouch, making it difficult to perform the usual lower uterine approach for a cesarian section; the baby had to be delivered through a fundal incision. Several methods for predicting intra-abdominal adhesions have been reported; however, in actual clinical practice, it is still difficult to diagnose all conditions in the abdominal cavity. Pregnancy with endometriosis increases the risk of perinatal complications, and management in tertiary centers should be considered. The prognosis of uterine fundal incision is unknown, and long-term follow-up of these cases will be required.

\section{Acknowledgements}

We would like to thank Editage (www.editage.com) for English language editing. 


\section{Author Contributions}

Yu Wakaki : She involved in the clinical care of the patient and contributed to the conception, drafting, review, and revision of the manuscript.

Kaori Watanabe : She involved in the clinical care of the patient and contributed to the conception and drafting.

Yukiko Kumasaka: She involved in the clinical care of the patient and contributed to the conception and drafting.

Rika Suzuki : She involved in the clinical care of the patient and contributed to the conception, drafting.

\section{References}

1. Visconti, F., Quaresima, P., Rania, E., et al. 2020. Difficult caesarean section: A literature review. European Journal of Obstetrics, Gynecology and Reproductive Biology 246:72-78.

2. Dogan, A., Ertas, I. E., Uyar, I., et al. 2016. Preoperative Association of Abdominal Striae Gravidarum with Intraabdominal Adhesions in Pregnant Women with a History of Previous Cesarean Section: a Crosssectional Study. Geburtshilfe und Frauenheilkd 76(3):268-272.

3. Çim, N., Elçi, E., Güneş Elçi, G., Almalı, N., Yıldızhan, R. 2018. Are the skin scar characteristics and closure of the parietal peritoneum associated with pelvic adhesions? Turkish Journal of Obstetrics and Gynecology 15(1):28-32.

4. Pergialiotis, V., Frountzas, M., Siotos, C., Karampetsou, N., Perrea, D. N., Efthymios Vlachos D. 2017. Cesarean wound scar characteristics for the prediction of pelvic adhesions: a meta-analysis of observational studies. The Journal of Maternal-Fetal \& Neonatal Medicine 30(4):486-491.

5. Taylan, E., Akdemir, A., Ergenoglu, A.M., Yeniel, A.O., Tekindal, M.A. 2017. Can We Predict the Presence and Severity of Intra-Abdominal Adhesions before Cesarean Delivery. Gynecologic and Obstetric Investigation 82(6):521-526.

6. Jaafar, Z. A. A., Obeid, R. Z., Salman, D. A. 2019. Skin markers and the prediction of intraabdominal adhesion during second Cesarean delivery. Ginekologia Polska 90(6):325-330.

7. Reid, S., Lu, C., Caiskar, I., et al. 2013. Prediction of pouch of Douglas obliteration in women with suspected endometriosis using a new real-time dynamic transvaginal ultrasound technique: the sliding sign. Ultrasound in Obstetrics \& Gynecology 41:685-691.

8. Reid, S., Lu, C., Caiskar, I., et al. 2013. The prediction of pouch of Douglas obliteration using offline analysis of the transvaginal ultrasound 'sliding sign' technique: inter- and intra-observer reproducibility. Human Reproduction 28(5):1237-1246.

9. Hundelist, G., Fritzer, N., Staettner, S., et al. 2013. Uterine sliding sign: a simple sonographic predictor for presence of deep infiltrating endometriosis of the rectum. Ultrasound in Obstetrics \& Gynecology 41(6):692-695.

10. Ichikawa, M., Akira, S., Kaseki, H., et al. 2020. Accuracy and clinical value of an adhesion scoring system: A preoperative diagnostic method using transvaginal ultrasonography for endometriotic adhesion. The Journal of Obstetrics and Gynaecology Research 46(3):466-478.

11. Baron, J., Tirosh, D., Mastrolia, S. A., et al. 2018. Sliding sign in third-trimester sonographic evaluation of intra-abdominal adhesions in women undergoing repeat Cesarean section: a novel technique. Ultrasound in Obstetrics \& Gynecology 52(5):662-665.

12. Shu, W. 2021. Predicting Intra-abdominal Adhesions for Repeat Cesarean Delivery with the Ultrasound Sliding Sign. Journal of Obstetrics and Gynaecology Canada 43(11):1274-1278. 
13. Zawin, M., McCarthy, S., Scoutt, L., Comite, F. 1989. Endometriosis: appearance and detection at MR imaging. Radiology 171(3):693-696.

14. Arrivé, L., Hricak, H., Martin, M. C. 1989. Pelvic endometriosis: MR imaging. Radiology 171(3):687-692.

15. Randall, D., Fenner, J., Gillott, R., et al. 2016. A novel diagnostic aid for detection of intra-abdominal adhesions to the anterior abdominal wall using dynamic magnetic resonance imaging. Gastroenterology Research and Practice 2016:2523768.

16. Randall, D., Joosten, F., Ten Broek, R. P., et al. 2017. A novel diagnostic aid for intra-abdominal adhesion detection in cine-MRI: pilot study and initial diagnostic impressions. The British Journal of Radiology 90(1077):20170158.

17. Sunkunami, K., Hattori, K., Nishijima, K., Kotsuji F. 2004. Transverse fundal uterine incision in a patient with placenta increra. The Journal of Maternal-Fetal \& Neonatal Medicine 16(6):355-356.

18. Kotsuji, F., Nishijima, K., Kurokawa, T., et al. 2013. Transverse uterine fundal incision for placenta praevia with accreta, involving the entire anterior uterine wall: a case series. BJOG 120(9):1144-1149.

19. Farland, L. V., Prescott, J., Sasamoto, N., et al. 2019. Endometriosis and Risk of Adverse Pregnancy Outcomes. Obstetrics and Gynecology 134(3):527-536.

20. Fujii, T., Wada-Hiraike, O., Nagamatsu, T., et al. 2016. Assisted reproductive technology pregnancy complications are significantly associated with endometriosis severity before conception: a retrospective cohort study. Reproductive Biology and Endocrinology 14(1):73.

21. Harada, T., Taniguchi, F., Amano, H., et al. 2019. Adverse obstetrical outcomes for women with endometriosis and adenomyosis: A large cohort of the Japan Environment and Children's Study. PLoS One 14(8):e0220256.

\section{Figure Legends}

\section{Figure 1}

a: MRI image performed at 30 weeks of gestational age

b: Position of the uterine walls during the operation. The bladder (shaded), anterior (crosses), fundus (horizontal stripes), and posterior (solid yellow) uterine walls are located at the front of the body.

\section{Figure 2}

a: The bladder is still firmly adhered to the anterior wall of the uterus after suturing of the uterine wound. A uterine incision extends towards the posterior wall of the uterus.

b: After the uterine suture, the incision (dotted line) crosses through the uterine fundus (solid line) toward the posterior wall. The round ligament (vertical stripe line) and oviduct (horizontal stripe lines) are located at the center, and the bladder (dotted pattern) is firmly adhered to the anterior uterine wall.

\section{Figure 3}

The posterior wall of the uterus adheres to the sigmoid colon, and lesions of endometriosis are apparent (white triangle).

\section{Figure 4}

Forty-one days after surgery, transvaginal ultrasound shows the wound (white triangle) located around the fundal of the uterus (solid white line). 

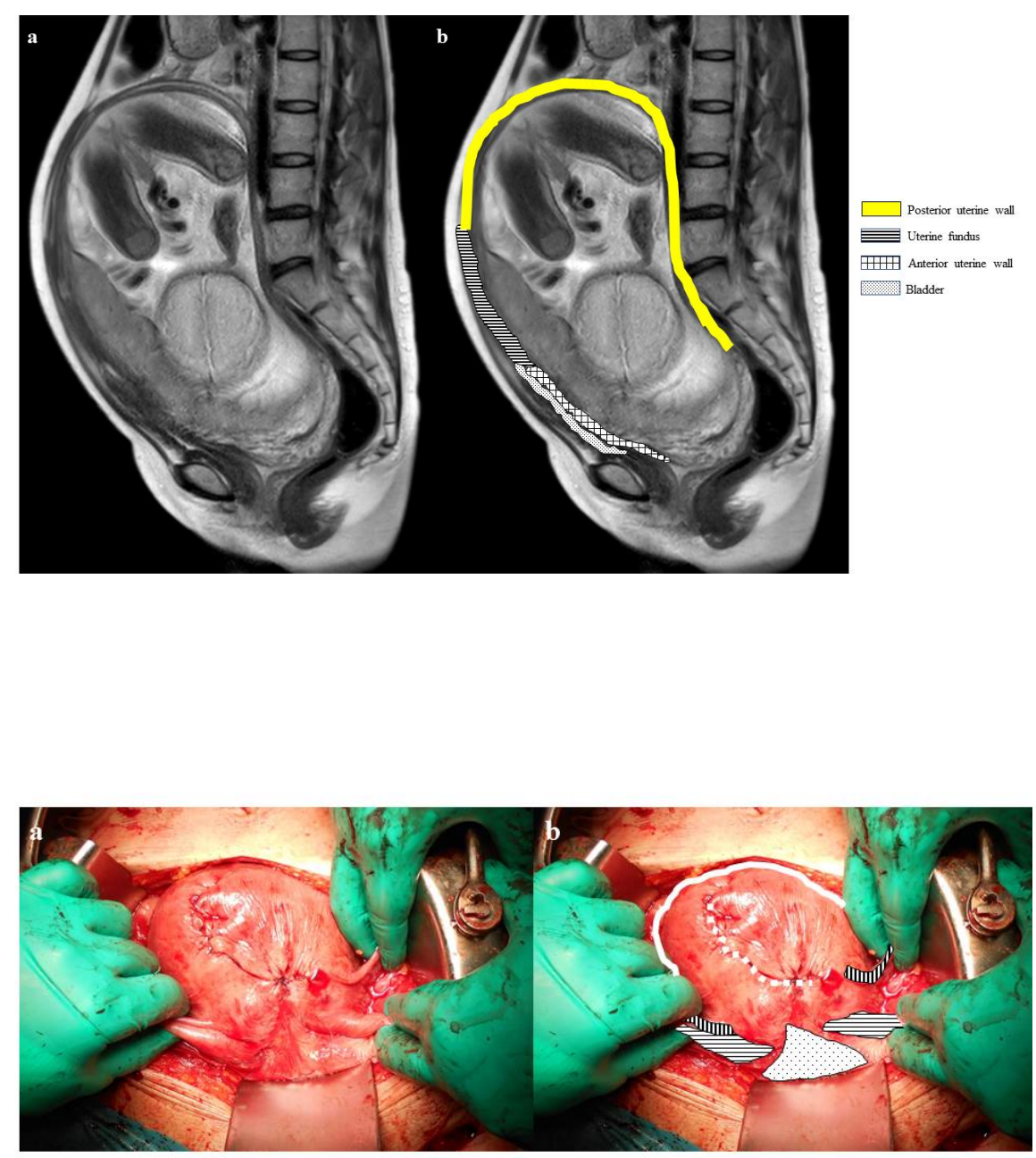

盂耑耑 Oviduct 

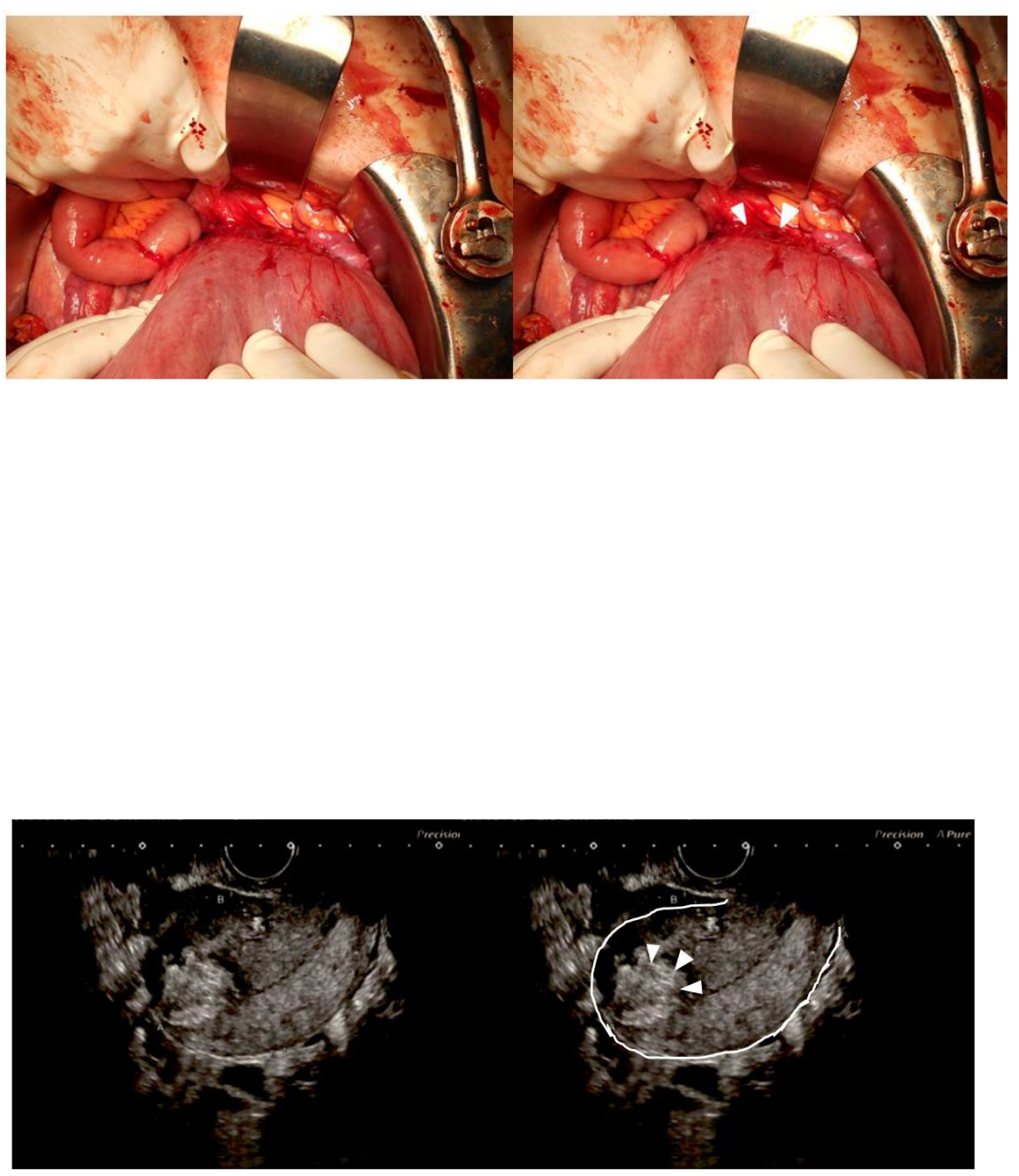